\title{
Global Health diplomacy for noncommunicable diseases prevention and control: a systematic review
}

\author{
Mahnaz Afshari ${ }^{1}$, Ahmad Ahmadi Teymourlouy ${ }^{1 *}$ (D), Mohsen Asadi-Lari ${ }^{2}$ and Mohammadreza Maleki ${ }^{1}$
}

\begin{abstract}
Introduction: The prevention and control of noncommunicable diseases (NCDs) are one of the main challenges of healthcare systems around the world. In addition to the technical level, it requires political negotiations and solutions, such as global health diplomacy (GHD), which involves the participation of a wide range of actors and stakeholders and innovative international health partnerships. This review aimed to draw lessons for strengthening linkages with a wide range of actors and stakeholders from the GHD literature for NCDs, and how policymakers and political leaders can effectively use international health partnerships to beat NCDs.
\end{abstract}

Methods: This research was a systematic review of the literature on GHD for NCDs. All relevant articles published in English were identified by searching PubMed, Web of Science, Scopus, and Embase databases, Google and Google Scholar search engines, and the reference lists of identified articles as well as a number of special journals. 30 articles that met the inclusion criteria were analyzed using content analysis in MAXQDA 10. The Global Health Diplomacy Pyramid and Blouin and Dubé's (2010) analytical framework for examining negotiations were used to classify the data.

Findings: 30 articles have been published on GHD for NCDs. Five key themes, i.e. the specific problem requiring global collective action, key actors, their interests in the problem, potential negotiation process, and potential scenarios for collective action and 46 sub-themes were identified. Moreover, given the importance of collaboration on NCDs in the international arena, actors were categorized into three groups based on the GHD Pyramid: (1) core diplomacy, (2) multi-stakeholder diplomacy, and (3) informal diplomacy.

Conclusion: Development and adoption of a global policy to tackle the rise in NCDs in developed and developing countries require policymakers and political leaders that participate in GHD. Successful developments in global health policy depend on the performance of and respectful relationships among the stakeholders, and global health diplomats need to understand the complexities of the institutional structures and functional relationships of the international institutions involved in health.

Keywords: Review [publication type], Diplomacy, Global Health, International cooperation, Noncommunicable diseases, Policymaking

\footnotetext{
*Correspondence: ahmadi.sa@iums.ac.ir

'Department of Health Service Management, School of Health Management and Information Sciences, Iran University of Medical Sciences, Tehran, Iran Full list of author information is available at the end of the article
}

(c) The Author(s). 2020 Open Access This article is licensed under a Creative Commons Attribution 4.0 International License, which permits use, sharing, adaptation, distribution and reproduction in any medium or format, as long as you give appropriate credit to the original author(s) and the source, provide a link to the Creative Commons licence, and indicate if changes were made. The images or other third party material in this article are included in the article's Creative Commons licence, unless indicated otherwise in a credit line to the material. If material is not included in the article's Creative Commons licence and your intended use is not permitted by statutory regulation or exceeds the permitted use, you will need to obtain permission directly from the copyright holder. To view a copy of this licence, visit http://creativecommons.org/licenses/by/4.0/ The Creative Commons Public Domain Dedication waiver (http://creativecommons.org/publicdomain/zero/1.0/) applies to the data made available in this article, unless otherwise stated in a credit line to the data. 


\section{Introduction}

Over the last two decades, global health issues have become more prominent in foreign policy. Events such as the HIV/AIDS pandemic, infectious diseases, the threat of bioterrorism, and issues related to trade and health have prompted policymakers to focus more on health issues [1-3]. As nations become more and more interconnected and as health-related issues increasingly become global issues, state actors in the domain of health are forced to seek cross-border collective action and collaboration with nonstate actors [2, 4]. United Nations (UN) General Assembly also emphasizes that governments must pay more attention to global health in their foreign policies and increase their negotiations and political interactions in this field [5-7].

Global health diplomacy (GHD) is the process of negotiated collective action for global health that can eventually lead to new forms of global health policy and governance [8]. This concept has received significant attention from key global health actors, including the World Health Organization (WHO), health and foreign affairs ministries of countries, and academia [9-13].

Although GHD has entered the mainstream of countries' foreign policy, it has different meanings, which could generally be divided into three categories based on the interaction of actors around global public health issues: (1) core diplomacy, i.e. formal negotiations among nations; (2) multi-stakeholder diplomacy, i.e. negotiations among nations and other actors that do not necessarily lead to agreements; and (3) informal diplomacy, i.e. interactions between international public health actors and their counterparts, including host country officials, nongovernmental organizations, private companies, and the public. Core and multi-stakeholder diplomacy in global health require the effective use of a delicate mix of technical expertise, legal knowledge, and diplomatic skills [14]. A successful global health strategy for addressing public health and foreign policy goals requires effective action at every level of the GHD pyramid, and GHD, as practiced by health attachés, entails the identification and engagement of key tools and actors as well as coordinated action by various counterparts and stakeholders [15].

Health diplomacy provides a political framework that aims to improve health in target populations and enhance governmental relations between collaborating countries. Governments offering health-related aid to a nation with which they wish to develop stronger diplomatic links have the advantage of developing a deeper relationship with its citizens [16]. This can be accomplished through different mechanisms such as providing general funding, ensuring the supply of essential drugs, investing in hospitals or equipment, and training health professionals. Significant investments have been made in
Sub-Saharan Africa, South America, and South-East Asia to eradicate specific diseases such as HIV/AIDS, malaria, and tuberculosis. However, there has been relatively little support for general health infrastructure, training, and education, or the burden of chronic diseases in developing countries [17].

Each year, 41 million people die from noncommunicable diseases (NCDs), which is equivalent to $71 \%$ of all deaths globally [18]. Evidence suggests the increasing burden of NCDs in low and middle-income countries (LMIC) $[19,20]$. NCDs in LMIC account for $80 \%$ of deaths and two-thirds of disabilities from NCDs worldwide. More specifically, NCDs in LMIC mostly affects people in their thirties, which is their most productive working years and this is serious threat to health and economic growth [21].

There is widespread international support for the fight against NCDs. The UN High-Level Meeting on Prevention and Control of NCDs [22], the WHO Global Action Plan for the Prevention and Control of NCDs 20132020 [23, 24], the WHO NCD Global Monitoring Framework [25], and recognition of NCDs as a major challenge to sustainable development [26] highlight this issue. Although such support for prevention and control of NCDs is encouraging, lack of international financing for these efforts necessitates the development of multistakeholder models to address the global burden of NCDs.

Prevention and control of NCDs is one of the key challenges of health systems that, in addition to a technical level, requires negotiations and political solutions such as global health diplomacy (GHD), which involves the participation of a wide range of actors and stakeholders [27]. Policy interventions for health, prevention, and control of NCDs must begin with diplomatic negotiations between state officials [28]. Health threats such as challenges to the safety of the global drug supply and the spread of chronic and NCDsthat impact national security increasingly highlight the need for diplomats who understand health issues and can negotiate effectively in the multinational foreign policy environment [27-29]. Insufficient focus on NCDs by different sectors at the national and international levels, including the lack of funding for NCD research, prevention, and control by governments and non-governmental organizations (NGOs) necessitate the development of coordinated strategies and diplomatic initiatives to address the multinational nature of this issue [30]. GHD for NCDs is defined as the process of negotiations by which state and nonstate actors attempt to develop and implement collective actions to solve global health challenges [1-4]. This paper argues that global health diplomacy and political interactions and partnerships between policy actors could contribute to a more effective global response and 
action to NCDs and leading to global health improvements. The purpose of this review is to draw lessons from the GHD literature for NCDs and how it can be effectively used by policymakers and political leaders.

\section{Methods}

This research ia a systematic review of the existing evidence on GHD for NCDs, including cardiovascular diseases, cancers, chronic respiratory diseases, and diabetes as well as their risk factors, i.e. tobacco use, excess salt and fat intake, alcohol abuse, and physical inactivity, in the period 2007-2019. The second stage involved content analysis of the studies with a qualitative approach.

To find relevant articles for this review, PubMed, Web of Science, Scopus, and Embase databases and Google and Google Scholar search engines were used. Keywords included $\mathrm{MeSH}$ and common terms related to the topic: "Diplomacy" OR "Internationality" OR "foreign policy" OR "foreign affairs" OR "international relations" OR "international politics" OR "statesmanship" OR "statecraft" OR "Health Diplomacy" OR "Medical Diplomacy" OR "Negotiations" OR "Multilateral Engagement" OR "Bilateral Agreements" AND "Drinking Behavior" "Alcoholic Beverages" OR "Smoking" OR "Smokers" OR "Feeding Behavior" OR "Diet" [MeSH] OR "Obesity" OR "Food" OR "Fast Foods" OR "Sugars" OR "Sodium, Dietary" OR "Exercise" [MeSH] OR "LifeStyle" OR "Healthy Lifestyle" OR "Sedentary Behavior" OR "Alcohol Drinking" $[\mathrm{MeSH}] \mathrm{OR}$ "Tobacco Use" OR "Tobacco Products" OR "Tobacco" OR "Noncommunicable Diseases". Moreover, the reference lists of identified articles were manually searched to find more relevant studies. The studies included in the qualitative synthesis include empirical studies, commentaries and review articles. Inclusion criteria for this study included literatures published in English language and the year of publication included studies published from 2007 to 2019 because initial searches of the literature showed that most relevant studies were conducted after 2007. Articles published in any language other than English, articles that were not unavailable in full text, dissertations, and redundant studies were excluded.

Overall, 1895 articles were extracted. First, the title and abstract of the articles were screened based on the inclusion criteria and Studies which did not address the research question and the duplicates of the same records were then excluded in this stage and leaving 48 articles for full-text review. 18 articles were excluded due to unavailability in full-text form or redundancy, and 30 articles were selected for the final review. The screening process and search results are provided in the PRISMA flow diagram [31] (Fig. 1). Data were collected using a data extraction form that was designed based on the objectives of the study. This form included entries on the author, publication year, country, method of research, key actors, content, context, Tools and main finding.

The remaining articles were entered into the quality assessment stage. Quality was assessed independently by two studies using the 15-point instrument of Mitton et al. [32]. Each item is given a score of 0 (not present or reported), 1 (present but low quality), 2 (present and midrange quality), or 3 (present and high quality). Criteria for quality assessment included: literature review and identification of research gaps; research questions, hypotheses, and design; population and sampling; data collection process and instruments; and analysis and reporting of results. Disagreements were resolved by discussion and, when essential, by consulting a third review

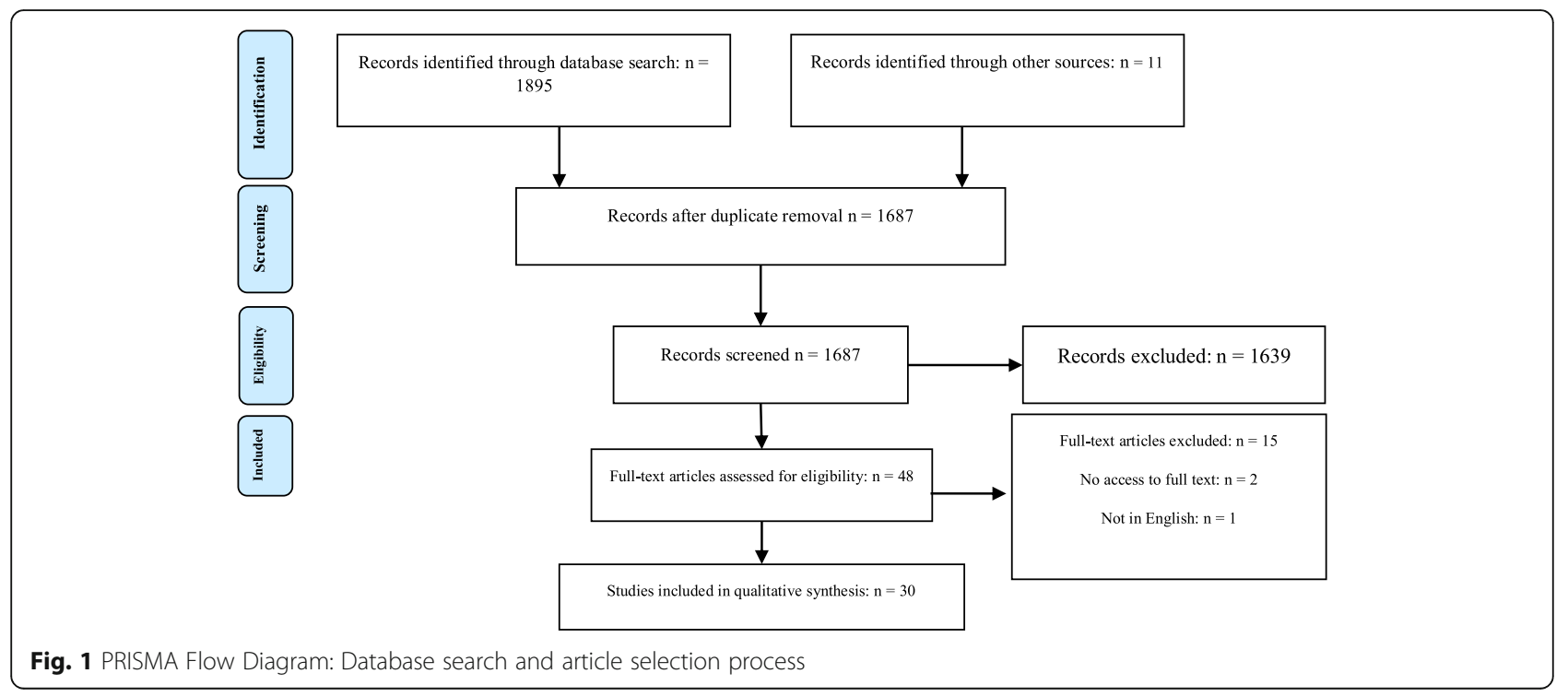


author. Given that the review was qualitative, articles were not removed at this stage, but more weight was given to articles with a quality rating of 10 or above in the data analysis and interpretation of results.

Then, a content analysis of the articles was performed. The data were coded and managed using MAXQDA 10 for Windows (VERBI GmbH, Berlin, Germany), and related themes and sub-themes for each article were extracted to identify the relationships among these themes and identify patterns and meanings. The GHD Pyramid $[14,15]$ and the Negotiations Review Analytical Framework [4] were used to classify the data.

\section{Results}

Between 2007 and 2019, 30 articles have been published on GHD for NCDs (Table 1). About $57 \%$ of the articles have been published since 2016, with the majority of studies (10 articles) published in 2018 (Fig. 2). Trends suggest that the number of articles has increased around 2011 and 2018 in which the UN General Assembly held high-level meetings on the prevention and control of NCDs.

In Table 2, five themes are identified using the Blouin and Dubé's (2010) analytical framework for examining negotiations: (1) the specific problem requiring global collective action, (2) key actors, (3) their interests in the problem, (4) potential negotiation process, and (5) potential scenarios for collective action. Besides, 46 subthemes related to GHD on NCDs are identified (Table 2).

Given the importance of mapping actors, their interests, and structural characteristics related to them, interactions in the international arena on NCDs are divided into three categories using the GHD Pyramid: (1) core diplomacy, (2) multi-stakeholder diplomacy, and (3) informal diplomacy. Actors, tools, contents, and contexts were extracted at each of these three levels (Table 3).

The key actors are identified at different levels of diplomacy: Core diplomacy: UN and associated agencies and groups, including the $\mathrm{WHO}$ and the Independent High-level Commission on NCDs, heads of government, international professional associations, International Diabetes Federation, Union for International Cancer Control, and World Heart Federation; Multi-stakeholder diplomacy: Asia-Pacific Economic Cooperation (APEC), national governments and ministries (e.g. health, foreign policy, education, finance, labor, etc.), commercial actors (e.g. pharmaceutical companies, a variety of businesses involved in the production, processing, distribution, marketing, and sales of food and beverages), civil society organizations and coalitions (e.g. Nutrition Foundation, National Center for Chronic Disease Prevention, National Cancer Institute), healthcare regulatory agencies, Parliamentary Committee for Prevention and Control of
NCDs, and National Inter-ministerial Tobacco Control Committee; Informal diplomacy: NCD Alliance (NCDA), Framework Convention on Tobacco Control (FCTC), and Union for International Cancer Control, research centers, the academia, the medical community, the private sector, patients and their families, international donors, and health advocacy groups, NGOs, and humanitarian organizations.

Critical information are discussed in the content of some declaration and agreement for raising global challenges to GHD and negotiation (Table 3). For example The Paris Declaration on Aid Effectiveness has a leading role in raising awareness of climate change on the international stage and advocating for strong climate action for new global challenges such as rising food and climate change and Port of Spain Summit Declaration helped to rise NCDs agenda in global setting.

\section{Discussion}

The purpose of this Systematic review was to draw lessons for strengthenning linkages with a wide range of actors and stakeholders from the GHD literature regarding the prevention and control of NCDs. GHD is focused on international negotiation that includes a wide range of processes, from finalizing agreements between multilateral or bilateral aid donors and recipient countries to the processes of making binding and nonbinding international agreements in health or related to health. Between 2007 and 2019, 30 articles have been published on GHD for NCDs.

Five themes, i.e. (1) the specific problem requiring global collective action, (2) key actors, (3) their interests in the problem, (4) potential negotiation process, and (5) potential scenarios for collective action and 41 subthemes on GHD in prevention and control of NCDs were identified. Moreover, given the importance of collaboration on NCDs in the international arena, actors were categorized into three groups based on the GHD Pyramid: (1) core diplomacy, (2) multi-stakeholder diplomacy, and (3) informal diplomacy.

Seven specific problems that require global collective action were identified: the globalization of marketing and advertising strategies, sanctions, multinational companies undermining the regulatory authority of national governments, the need for stronger engagement with developing countries for GHD on obesity and tobacco control, current migration and travel patterns, trafficking, and trade liberalization. International commitments have changed the dynamics of countries' domestic policies. For example, multinational tobacco companies undermine the regulatory authority of national governments through public relations and lobbying strategies [61]. Due to the asymmetry of resources between large global tobacco firms and the governments of small countries, 
Table 1 Characteristics of studies on GHD for NCDs

\begin{tabular}{|c|c|c|c|c|c|c|c|c|}
\hline Author & $\begin{array}{l}\text { Country/ } \\
\text { Region }\end{array}$ & Year & Journal & $\begin{array}{l}\text { Level of } \\
\text { Diplomacy }\end{array}$ & Domain & article type & Method & key findings \\
\hline $\begin{array}{l}\text { Hospedales et al. } \\
\text { [33] }\end{array}$ & CARICOM* & 2011 & $\begin{array}{l}\text { Pan American } \\
\text { Journal of } \\
\text { Public Health }\end{array}$ & Regional & $\begin{array}{l}\text { Advocacy, Political } \\
\text { Factors }\end{array}$ & $\begin{array}{l}\text { Commentary } \\
\text { and } \\
\text { perspectives }\end{array}$ & $\begin{array}{l}\text { Qualitative- opinion } \\
\text { piece on the process } \\
\text { of and lessons } \\
\text { learned in achieving } \\
\text { the political } \\
\text { commitment of } \\
\text { heads of } \\
\text { government } \\
\text { manifested by a one- } \\
\text { day summit on the } \\
\text { prevention and con- } \\
\text { trol of NCD** }\end{array}$ & $\begin{array}{l}\text { Implementation of } \\
\text { the NCD Summit } \\
\text { Declaration mandates } \\
\text { was most effective in } \\
\text { larger countries with } \\
\text { greater capacity, but } \\
\text { countries of all sizes } \\
\text { performed well, } \\
\text { when they had } \\
\text { regional or global } \\
\text { support. Progress was } \\
\text { limited in regional } \\
\text { approaches to food } \\
\text { security, labeling, and } \\
\text { elimination of trans } \\
\text { fats. Inadequate } \\
\text { funding stymied } \\
\text { several resource- } \\
\text { dependent interven- } \\
\text { tions. Monitoring } \\
\text { mechanisms were } \\
\text { established, but more } \\
\text { concrete goals are } \\
\text { needed, especially for } \\
\text { actions of non-health } \\
\text { government } \\
\text { agencies. }\end{array}$ \\
\hline $\begin{array}{l}\text { Lencucha et al. } \\
\text { [34] }\end{array}$ & Canada & 2010 & $\begin{array}{l}\text { Health Policy } \\
\text { and Planning }\end{array}$ & Global & $\begin{array}{l}\text { NGOs, GHD, FCTC, } \\
\text { International } \\
\text { Negotiations, } \\
\text { Global Health } \\
\text { Governance }\end{array}$ & $\begin{array}{l}\text { Original } \\
\text { study }\end{array}$ & $\begin{array}{l}\text { Grounded theory- } \\
\text { qualitative data from } \\
\text { public documents } \\
\text { and in-depth inter- } \\
\text { views with partici- } \\
\text { pants from the } \\
\text { government and } \\
\text { NGOs* }\end{array}$ & $\begin{array}{l}\text { Five key activities or } \\
\text { roles of the Canadian } \\
\text { NGOs during the } \\
\text { negotiation of the } \\
\text { FCTC*: monitoring, }_{\text {lobbying, brokering }} \\
\text { knowledge, offering } \\
\text { technical expertise } \\
\text { and fostering } \\
\text { inclusion. }\end{array}$ \\
\hline Blouin [30] & - & 2012 & $\begin{array}{l}\text { Administrative } \\
\text { Sciences }\end{array}$ & Global & $\begin{array}{l}\text { Global Health, } \\
\text { Diplomacy, NCDs, } \\
\text { Chronic Diseases, } \\
\text { Policy, Global } \\
\text { Collective Action }\end{array}$ & Review & $\begin{array}{l}\text { Review_social } \\
\text { sciences literature on } \\
\text { policymaking at the } \\
\text { domestic and } \\
\text { international level }\end{array}$ & $\begin{array}{l}\text { Adopting a global } \\
\text { strategy with partners } \\
\text { to increase media } \\
\text { coverage. } \\
\text { key role of civil } \\
\text { society organizations } \\
\text { in a strong collective } \\
\text { response }\end{array}$ \\
\hline Lee [35] & Brazil & 2010 & PLoS Medicine & $\begin{array}{l}\text { National/ } \\
\text { Global }\end{array}$ & $\begin{array}{l}\text { NGOs, GHD*, FCTC, } \\
\text { International } \\
\text { Negotiations }\end{array}$ & $\begin{array}{l}\text { Original } \\
\text { study }\end{array}$ & $\begin{array}{l}\text { key informant } \\
\text { interviews with } \\
\text { Brazilian } \\
\text { policymakers, } \\
\text { diplomats, and } \\
\text { public health } \\
\text { advocates on the } \\
\text { country's role in } \\
\text { FCTC negotiations, } \\
\text { literature review of } \\
\text { priary and secondary } \\
\text { sources }\end{array}$ & $\begin{array}{l}\text { providing leadership } \\
\text { throughout the } \\
\text { negotiation process }\end{array}$ \\
\hline $\begin{array}{l}\text { Blouin \& Dubé } \\
\text { [36] }\end{array}$ & - & 2010 & $\begin{array}{l}\text { Journal of } \\
\text { Public Health } \\
\text { Policy }\end{array}$ & Global & $\begin{array}{l}\text { Obesity } \\
\text { Prevention, NGOs, } \\
\text { GHD, FCTC, } \\
\text { International } \\
\text { Negotiations }\end{array}$ & Review & $\begin{array}{l}\text { Review of } \\
\text { documents and } \\
\text { studies - a simple } \\
\text { analytical framework } \\
\text { is used: (1) the } \\
\text { specific problem } \\
\text { requiring global } \\
\text { collective action, (2) }\end{array}$ & $\begin{array}{l}\text { require a much } \\
\text { stronger } \\
\text { engagement with } \\
\text { developing countries }\end{array}$ \\
\hline
\end{tabular}


Table 1 Characteristics of studies on GHD for NCDs (Continued)

\begin{tabular}{|c|c|c|c|c|c|c|c|c|}
\hline Author & $\begin{array}{l}\text { Country/ } \\
\text { Region }\end{array}$ & Year & Journal & $\begin{array}{l}\text { Level of } \\
\text { Diplomacy }\end{array}$ & Domain & article type & Method & key findings \\
\hline & & & & & & & $\begin{array}{l}\text { key actors, (3) their } \\
\text { interests in the } \\
\text { problem, (4) } \\
\text { potential negotiation } \\
\text { process, and (5) } \\
\text { potential scenarios } \\
\text { for collective action }\end{array}$ & \\
\hline $\begin{array}{l}\text { Mamudu \& } \\
\text { Glantz [37] }\end{array}$ & - & 2009 & $\begin{array}{l}\text { Global Public } \\
\text { Health }\end{array}$ & Global & $\begin{array}{l}\text { Civil Society, GHD, } \\
\text { FCTC, International } \\
\text { Negotiations }\end{array}$ & $\begin{array}{l}\text { Original } \\
\text { study }\end{array}$ & $\begin{array}{l}\text { Interview and } \\
\text { triangulation of } \\
\text { archival documents } \\
\text { and }\end{array}$ & $\begin{array}{l}\text { Using proper } \\
\text { strategies including } \\
\text { publication of a } \\
\text { newsletter, shaming, } \\
\text { symbolism and } \\
\text { media advocacy by } \\
\text { the FCA to influence } \\
\text { policy positions of } \\
\text { countries during the } \\
\text { FCTC negotiation. }\end{array}$ \\
\hline $\begin{array}{l}\text { Teixeira et al. } \\
\text { [38] }\end{array}$ & Brazil & 2017 & $\begin{array}{l}\text { Cadernos de } \\
\text { Saúde Pública }\end{array}$ & $\begin{array}{l}\text { National/ } \\
\text { Global }\end{array}$ & $\begin{array}{l}\text { Civil Society, GHD, } \\
\text { FCTC, International } \\
\text { Negotiations }\end{array}$ & $\begin{array}{l}\text { Original } \\
\text { study }\end{array}$ & $\begin{array}{l}\text { Kingdon's } \\
\text { perspective }\end{array}$ & $\begin{array}{l}\text { The link between } \\
\text { tobacco-related } \\
\text { healthcare measures } \\
\text { by technically skilled } \\
\text { officials, the involve- } \\
\text { ment of the high } \\
\text { echelon of the Minis- } \\
\text { try of Foreign Affairs } \\
\text { (policy flow), the ini- } \\
\text { tiative for the estab- } \\
\text { lishment of the } \\
\text { WHO*-FCTC (problem } \\
\text { flow), and the exist- } \\
\text { ence of a favorable } \\
\text { environment in ex- } \\
\text { ecutive and legisla- } \\
\text { tive (political flow), } \\
\text { opened a opportun- } \\
\text { ity window for WHO- } \\
\text { FCTC approval and } \\
\text { its inclusion in the } \\
\text { government decision } \\
\text { agenda. }\end{array}$ \\
\hline Sener [39] & - & 2014 & $\begin{array}{l}\text { American } \\
\text { Journal of } \\
\text { Surgery }\end{array}$ & Global & $\begin{array}{l}\text { Medical } \\
\text { Diplomacy, } \\
\text { Tobacco Control, } \\
\text { Breast Health }\end{array}$ & $\begin{array}{l}\text { Commentary } \\
\text { and } \\
\text { perspectives }\end{array}$ & Qualitative & $\begin{array}{l}\text { Unprecedented } \\
\text { presence and } \\
\text { participation of NGOs } \\
\text { in the drafting stages } \\
\text { was helpful for } \\
\text { starting negotiations } \\
\text { for the treaty }\end{array}$ \\
\hline $\begin{array}{l}\text { Smith \& Irwin } \\
\text { [40] }\end{array}$ & India & 2016 & $\begin{array}{l}\text { Globalization } \\
\text { and Health }\end{array}$ & $\begin{array}{l}\text { National/ } \\
\text { Global }\end{array}$ & $\begin{array}{l}\text { Food and Non- } \\
\text { alcoholic Bever- } \\
\text { ages to Children, } \\
\text { GHD, International } \\
\text { Negotiations }\end{array}$ & $\begin{array}{l}\text { Commentary } \\
\text { and } \\
\text { perspectives }\end{array}$ & $\begin{array}{l}\text { Ethnographic study, } \\
\text { in-depth interviews }\end{array}$ & $\begin{array}{l}\text { a successful GHD in } \\
\text { international level is } \\
\text { part of a process, not } \\
\text { the end, and an } \\
\text { important part of } \\
\text { conducting and } \\
\text { evaluating GHD is a } \\
\text { consideration of } \\
\text { challenges and } \\
\text { barriers concerning } \\
\text { national action. }\end{array}$ \\
\hline $\begin{array}{l}\text { Pearlman et al. } \\
\text { [21] }\end{array}$ & - & 2016 & $\begin{array}{l}\text { Science \& } \\
\text { Diplomacy }\end{array}$ & Global & $\begin{array}{l}\text { GHD, Cancer, } \\
\text { Multi-stakeholder } \\
\text { collaborations, } \\
\text { NCDs }\end{array}$ & case study & $\begin{array}{l}\text { Document review } \\
\text { and evidence-based } \\
\text { survey }\end{array}$ & $\begin{array}{l}\text { overcome many } \\
\text { barriers with Effective } \\
\text { partnership and } \\
\text { careful coordination }\end{array}$ \\
\hline $\begin{array}{l}\text { Wipfli \& Samet } \\
\text { [41] }\end{array}$ & - & 2012 & $\begin{array}{l}\text { Tobacco } \\
\text { Control }\end{array}$ & Global & $\begin{array}{l}\text { GHD, FCTC, } \\
\text { International }\end{array}$ & $\begin{array}{l}\text { Commentary } \\
\text { and }\end{array}$ & $\begin{array}{l}\text { Extending the } \\
\text { lessons learned from }\end{array}$ & $\begin{array}{l}\text { The collective } \\
\text { response to NCDs }\end{array}$ \\
\hline
\end{tabular}


Table 1 Characteristics of studies on GHD for NCDs (Continued)

\begin{tabular}{|c|c|c|c|c|c|c|c|c|}
\hline Author & $\begin{array}{l}\text { Country/ } \\
\text { Region }\end{array}$ & Year & Journal & $\begin{array}{l}\text { Level of } \\
\text { Diplomacy }\end{array}$ & Domain & article type & Method & key findings \\
\hline & & & & & Negotiations & perspectives & $\begin{array}{l}\text { tobacco control to } \\
\text { NCD control }\end{array}$ & $\begin{array}{l}\text { should centralize on } \\
\text { generating } \\
\text { multisectoral } \\
\text { evidence about the } \\
\text { transnational factors } \\
\text { influencing the rise in } \\
\text { NCDs and their risk } \\
\text { factors. }\end{array}$ \\
\hline Juma et al. [42] & $\begin{array}{l}\text { Kenya, } \\
\text { South } \\
\text { Africa, } \\
\text { Cameroon, } \\
\text { Nigeria, } \\
\text { and Malawi }\end{array}$ & 2018 & $\begin{array}{l}\text { BMC Public } \\
\text { Health }\end{array}$ & $\begin{array}{l}\text { National/ } \\
\text { Regional }\end{array}$ & $\begin{array}{l}\text { Multi-sectoral } \\
\text { Action }\end{array}$ & $\begin{array}{l}\text { multiple case } \\
\text { study }\end{array}$ & $\begin{array}{l}\text { Document reviews } \\
\text { and key informant } \\
\text { interviews, } \\
\text { McQueen's } \\
\text { framework for } \\
\text { intersectoral } \\
\text { governance }\end{array}$ & $\begin{array}{l}\text { The need for } \\
\text { mechanisms } \\
\text { including approaches } \\
\text { to capacity building } \\
\text { and resource } \\
\text { production to be } \\
\text { able to take multi- } \\
\text { sectoral measures in } \\
\text { policy development, } \\
\text { implementation and } \\
\text { monitoring of NCD } \\
\text { results. }\end{array}$ \\
\hline Dain [43] & - & 2018 & $\begin{array}{l}\text { International } \\
\text { Journal of } \\
\text { Health Policy } \\
\text { and } \\
\text { Management }\end{array}$ & Global & $\begin{array}{l}\text { Civil Society } \\
\text { Networks, Coalition } \\
\text { Building, Advocacy, } \\
\text { Governance, } \\
\text { Human Sustainable } \\
\text { Development }\end{array}$ & $\begin{array}{l}\text { Commentary } \\
\text { and } \\
\text { perspectives }\end{array}$ & $\begin{array}{l}\text { Shiffman's 'Four } \\
\text { Challenges that } \\
\text { Global Health } \\
\text { Networks Face' }\end{array}$ & $\begin{array}{l}\text { NCDA's* advocacy } \\
\text { has contributed to } \\
\text { the call for global } \\
\text { political } \\
\text { commitment. }\end{array}$ \\
\hline Russell et al. [44] & - & 2018 & $\begin{array}{l}\text { Global Public } \\
\text { Health }\end{array}$ & Global & $\begin{array}{l}\text { GHD, E-cigarettes, } \\
\text { FCTC, International } \\
\text { Law }\end{array}$ & $\begin{array}{l}\text { Original } \\
\text { study }\end{array}$ & Ethnography method & $\begin{array}{l}\text { One of the important } \\
\text { tasks of the FCA is to } \\
\text { prepare policy brief } \\
\text { on key issues. }\end{array}$ \\
\hline Frech [45] & $\begin{array}{l}\text { Latin } \\
\text { America }\end{array}$ & 2018 & $\begin{array}{l}\text { Journal of } \\
\text { Global } \\
\text { Oncology }\end{array}$ & $\begin{array}{l}\text { Regional/ } \\
\text { Global }\end{array}$ & $\begin{array}{l}\text { Cancer Research } \\
\text { and Control, } \\
\text { Partnerships and } \\
\text { Diplomacy }\end{array}$ & $\begin{array}{l}\text { Commentary } \\
\text { and } \\
\text { perspectives }\end{array}$ & Qualitative & $\begin{array}{l}\text { The need for high- } \\
\text { level cooperation } \\
\text { (the President's com- } \\
\text { mitment), the sharing } \\
\text { of information to } \\
\text { support the efficient } \\
\text { use of limited re- } \\
\text { sources, the preven- } \\
\text { tion of repeated } \\
\text { attempts, and the } \\
\text { synergy of current in- } \\
\text { vestments in specific } \\
\text { countries are } \\
\text { essential. }\end{array}$ \\
\hline Beaglehole [46] & - & 2011 & Lancet & Global & $\begin{array}{l}\text { UN* High-Level } \\
\text { Meeting on NCDs, } \\
\text { Global Crisis, Need } \\
\text { for Global Re- } \\
\text { sponse, Inter- } \\
\text { national Cooper- } \\
\text { ation, Monitoring } \\
\text { and Accountability }\end{array}$ & $\begin{array}{l}\text { Commentary } \\
\text { and } \\
\text { perspectives }\end{array}$ & $\begin{array}{l}\text { Report- providing } \\
\text { evidence for the } \\
\text { realities of the NCD } \\
\text { situation, summaries } \\
\text { key messages for } \\
\text { heads of state and } \\
\text { governments. }\end{array}$ & $\begin{array}{l}\text { Long-term success } \\
\text { requires inspiring and } \\
\text { committed national } \\
\text { and international } \\
\text { leadership, improving } \\
\text { primary health care, } \\
\text { effective use of } \\
\text { existing resources, } \\
\text { new financing } \\
\text { methods. }\end{array}$ \\
\hline Nishtar et al. [47] & - & 2018 & Lancet & Global & $\begin{array}{l}\text { WHO Independent } \\
\text { High-level Com- } \\
\text { mission on NCDs, } \\
\text { National Response } \\
\text { to NCDs, Inter- } \\
\text { national } \\
\text { Cooperation }\end{array}$ & $\begin{array}{l}\text { Commentary } \\
\text { and } \\
\text { perspectives }\end{array}$ & $\begin{array}{l}\text { This report } \\
\text { represented rich and } \\
\text { diverse views and } \\
\text { perspectives. }\end{array}$ & $\begin{array}{l}\text { Ensureing } \\
\text { implementation } \\
\text { through legislation, } \\
\text { regulation and } \\
\text { standards or } \\
\text { investment. } \\
\text { Health in all policies, } \\
\text { approaches of the } \\
\text { whole government, } \\
\text { the whole society }\end{array}$ \\
\hline
\end{tabular}


Table 1 Characteristics of studies on GHD for NCDs (Continued)

\begin{tabular}{|c|c|c|c|c|c|c|c|c|}
\hline Author & $\begin{array}{l}\text { Country/ } \\
\text { Region }\end{array}$ & Year & Journal & $\begin{array}{l}\text { Level of } \\
\text { Diplomacy }\end{array}$ & Domain & article type & Method & key findings \\
\hline & & & & & & & & $\begin{array}{l}\text { and intersectoral } \\
\text { approaches must be } \\
\text { taken in the field of } \\
\text { NCDs actions. Need } \\
\text { technical support, } \\
\text { training, practical } \\
\text { research and capacity } \\
\text { building initiatives. }\end{array}$ \\
\hline $\begin{array}{l}\text { Samuels \& } \\
\text { Hospedales [48] }\end{array}$ & CARICOM & 2011 & $\begin{array}{l}\text { West Indian } \\
\text { Medical } \\
\text { Journal }\end{array}$ & $\begin{array}{l}\text { Regional/ } \\
\text { Global }\end{array}$ & $\begin{array}{l}\text { Heads of } \\
\text { government, UN } \\
\text { High-Level Meet- } \\
\text { ing on NCDs }\end{array}$ & $\begin{array}{l}\text { Commentary } \\
\text { and } \\
\text { perspectives }\end{array}$ & Report & $\begin{array}{l}\text { commit to } \\
\text { strengthening } \\
\text { systems and incrising } \\
\text { resources, endorse } \\
\text { and implement the } \\
\text { commitments made } \\
\text { and identify and } \\
\text { support leadership } \\
\text { for sustained action } \\
\text { and accountability for } \\
\text { these initiatives. }\end{array}$ \\
\hline Hatefi et al. [49] & - & 2018 & $\begin{array}{l}\text { Bulletin of the } \\
\text { World Health } \\
\text { Organization }\end{array}$ & Global & $\begin{array}{l}\text { Global } \\
\text { Susceptibility to } \\
\text { NCDs, } \\
\text { Accountability }\end{array}$ & $\begin{array}{l}\text { Commentary } \\
\text { and } \\
\text { perspectives }\end{array}$ & $\begin{array}{l}\text { Perspectives on } \\
\text { rational response to } \\
\text { global health risks }\end{array}$ & $\begin{array}{l}\text { The main response } \\
\text { to NCDs must } \\
\text { happen downstream } \\
\text { at the country level. }\end{array}$ \\
\hline $\begin{array}{l}\text { Wickramasinghe } \\
\text { et al. [50] }\end{array}$ & $\begin{array}{l}\text { Lebanon, } \\
\text { Morocco, } \\
\text { Sudan, and } \\
\text { Yemen }\end{array}$ & 2018 & $\begin{array}{l}\text { Global Health } \\
\text { Action }\end{array}$ & $\begin{array}{l}\text { National/ } \\
\text { Regional }\end{array}$ & $\begin{array}{l}\text { Multisectoral } \\
\text { Action, National- } \\
\text { level stakeholders }\end{array}$ & original study & $\begin{array}{l}\text { Structured interviews } \\
\text { with key } \\
\text { stakeholders }\end{array}$ & $\begin{array}{l}\text { Achievement to } \\
\text { national multisectoral } \\
\text { action plans } \\
\text { development } \\
\text { through collaboration } \\
\text { and good technical } \\
\text { support. }\end{array}$ \\
\hline Mendis [51] & - & 2010 & $\begin{array}{l}\text { British Medical } \\
\text { Bulletin }\end{array}$ & Global & $\begin{array}{l}\text { Policies to Support } \\
\text { Regulatory, } \\
\text { Legislative, } \\
\text { Intersectoral } \\
\text { Action }\end{array}$ & $\begin{array}{l}\text { Commentary } \\
\text { and } \\
\text { perspectives }\end{array}$ & Qualitative review & $\begin{array}{l}\text { Needed to develop } \\
\text { innovative } \\
\text { approaches for } \\
\text { revenue generation } \\
\text { for prevention and } \\
\text { control of NCDs. } \\
\text { Adapted agenda } \\
\text { concering the } \\
\text { context of contries. }\end{array}$ \\
\hline $\begin{array}{l}\text { Maher \& Sridhar } \\
\text { [52] }\end{array}$ & - & 2012 & $\begin{array}{l}\text { Journal of } \\
\text { Global Health }\end{array}$ & Global & $\begin{array}{l}\text { Global Fight } \\
\text { Against NCDs, } \\
\text { global health } \\
\text { policy } \\
\text { communities, } \\
\text { political leaders }\end{array}$ & $\begin{array}{l}\text { discipline } \\
\text { configurative } \\
\text { case study }\end{array}$ & $\begin{array}{l}\text { Qualitative } \\
\text {-Shiffman's } 2009 \\
\text { political priority } \\
\text { framework }\end{array}$ & $\begin{array}{l}\text { Engaging the diverse } \\
\text { actors for the global } \\
\text { proliferation of NCDs. }\end{array}$ \\
\hline $\begin{array}{l}\text { Gneiting \& } \\
\text { Schmitz [53] }\end{array}$ & - & 2016 & $\begin{array}{l}\text { Health Policy } \\
\text { and Planning }\end{array}$ & Global & $\begin{array}{l}\text { Advocacy, Political } \\
\text { Factors, Network } \\
\text { Formation and } \\
\text { Evolution in } \\
\text { International } \\
\text { Health Governance }\end{array}$ & $\begin{array}{l}\text { Original } \\
\text { study }\end{array}$ & $\begin{array}{l}\text { In-depth qualitative } \\
\text { analysis, in-depth } \\
\text { examination of social } \\
\text { and political pro- } \\
\text { cesses with a paired } \\
\text { comparison }\end{array}$ & $\begin{array}{l}\text { global health } \\
\text { networks (individuals } \\
\text { to a global coalition } \\
\text { of membership) are } \\
\text { engaging in } \\
\text { advocacy on a given } \\
\text { health problem. }\end{array}$ \\
\hline Magnusson [54] & - & 2007 & $\begin{array}{l}\text { Globalization } \\
\text { and Health }\end{array}$ & Global & $\begin{array}{l}\text { Global Health } \\
\text { Governance }\end{array}$ & $\begin{array}{l}\text { Commentary } \\
\text { and } \\
\text { perspectives }\end{array}$ & Report & $\begin{array}{l}\text { Needed to broader } \\
\text { framework of } \\
\text { reference for lifestyle- } \\
\text { related NCDs }\end{array}$ \\
\hline $\begin{array}{l}\text { Battams \& } \\
\text { Townsend [55] }\end{array}$ & - & 2018 & $\begin{array}{l}\text { Critical Public } \\
\text { Health }\end{array}$ & $\begin{array}{l}\text { Global/ } \\
\text { nation }\end{array}$ & $\begin{array}{l}\text { trade policy, policy } \\
\text { coherence, social } \\
\text { determinants of } \\
\text { health, advocacy }\end{array}$ & original study & $\begin{array}{l}\text { Interviews with key } \\
\text { actors working } \\
\text { across trade and } \\
\text { health sectors }\end{array}$ & $\begin{array}{l}\text { Support for advocacy } \\
\text { coalitions operating } \\
\text { basedn upon trade } \\
\text { and geopolitical } \\
\text { interests. } \\
\text { Lobbying trade } \\
\text { policy actors }\end{array}$ \\
\hline
\end{tabular}


Table 1 Characteristics of studies on GHD for NCDs (Continued)

\begin{tabular}{|c|c|c|c|c|c|c|c|c|}
\hline Author & $\begin{array}{l}\text { Country/ } \\
\text { Region }\end{array}$ & Year & Journal & $\begin{array}{l}\text { Level of } \\
\text { Diplomacy }\end{array}$ & Domain & article type & Method & key findings \\
\hline & & & & & & & & $\begin{array}{l}\text { proactively and } \\
\text { benefit from linking } \\
\text { with global advocacy } \\
\text { networks as a way to } \\
\text { counter the power } \\
\text { and resources of } \\
\text { industries with NCD } \\
\text { risk areas. }\end{array}$ \\
\hline Kirton et al. [56] & CARICOM & 2018 & $\begin{array}{l}\text { Pan American } \\
\text { Journal of } \\
\text { Public Health }\end{array}$ & $\begin{array}{l}\text { Regional/ } \\
\text { global }\end{array}$ & $\begin{array}{l}\text { Port of Spain } \\
\text { Summit } \\
\text { Declaration, global } \\
\text { and regional } \\
\text { action }\end{array}$ & $\begin{array}{l}\text { Original } \\
\text { study }\end{array}$ & $\begin{array}{l}\text { Using data from } \\
\text { published literature, } \\
\text { primary documents, } \\
\text { and semistructured } \\
\text { interviews (a method } \\
\text { developed by the } \\
\text { University of } \\
\text { Toronto's Global } \\
\text { Governance } \\
\text { Program) }\end{array}$ & $\begin{array}{l}\text { requirement to } \\
\text { embed NCDs in a } \\
\text { whole-of-global-gov- } \\
\text { ernance approach, } \\
\text { monitor implementa- } \\
\text { tion annually, de- } \\
\text { velop transregional } \\
\text { partnerships, engage } \\
\text { civil society and sup- } \\
\text { port regular regional } \\
\text { and global summits }\end{array}$ \\
\hline $\begin{array}{l}\text { Greaves et al. } \\
\text { [57] }\end{array}$ & CARICOM & 2018 & $\begin{array}{l}\text { Pan American } \\
\text { Journal of } \\
\text { Public Health }\end{array}$ & Regional & $\begin{array}{l}\text { Port of Spain } \\
\text { Summit } \\
\text { Declaration, Health } \\
\text { communication }\end{array}$ & $\begin{array}{l}\text { Commentary } \\
\text { and } \\
\text { perspectives }\end{array}$ & Report and review & $\begin{array}{l}\text { the NCDs advocate } \\
\text { should be considered } \\
\text { as knowledge broker } \\
\text { performing tasks } \\
\text { related to effective } \\
\text { knowledge transfer, } \\
\text { networking and } \\
\text { capacity building }\end{array}$ \\
\hline Chattu et al. [58] & CARICOM & 2019 & $\begin{array}{l}\text { Health } \\
\text { Promotion } \\
\text { Perspectives }\end{array}$ & Global & $\begin{array}{l}\text { Port of Spain } \\
\text { Summit } \\
\text { Declaration, } \\
\text { outcome of GHD }\end{array}$ & review & systematic review & $\begin{array}{l}\text { Impact of the NCDs } \\
\text { regional summit } \\
\text { declaration on global } \\
\text { attention to the } \\
\text { Prevention and } \\
\text { Control of NCDs. }\end{array}$ \\
\hline $\begin{array}{l}\text { McBride et al. } \\
\text { [59] }\end{array}$ & - & 2019 & $\begin{array}{l}\text { BMC Public } \\
\text { Health }\end{array}$ & Global & $\begin{array}{l}\text { GHD, Soft power, } \\
\text { Global health } \\
\text { agenda-setting, } \\
\text { SDGs**, BRICS, G7, } \\
\text { G20 }\end{array}$ & $\begin{array}{l}\text { Original } \\
\text { study }\end{array}$ & $\begin{array}{l}\text { Content analysis to } \\
\text { review the health } \\
\text { ministerial } \\
\text { communiqués issued } \\
\text { by the political clubs } \\
\text { after the SDGs were } \\
\text { adopted at the UN } \\
\text { General Assembly of } \\
\text { September } 2015\end{array}$ & $\begin{array}{l}\text { The global health } \\
\text { leadership of the } \\
\text { BRICS, G7 and G20 } \\
\text { represents an } \\
\text { exercise of soft } \\
\text { power and GHD on } \\
\text { NCDs and their risk } \\
\text { factors. }\end{array}$ \\
\hline Collins et al. [60] & - & 2019 & The bmj & Global & $\begin{array}{l}\text { development } \\
\text { cooperation, } \\
\text { global action, }\end{array}$ & $\begin{array}{l}\text { Commentary } \\
\text { and } \\
\text { perspectives }\end{array}$ & Report and review & $\begin{array}{l}\text { Development South- } \\
\text { South and triangular } \\
\text { cooperation beyond } \\
\text { North-South develop- } \\
\text { ment assistance. }\end{array}$ \\
\hline
\end{tabular}

* noncommunicable diseases (NCDs), global health diplomacy (GHD), United Nations (UN), World Health Organization (WHO), non-governmental organizations (NGOs), The Caribbean Community (CARICOM), Framework Convention on Tobacco Control (FCTC), noncommunicable diseases alliance (NCDA), Sustainable development goals (SDGs)

this problem is specifically acute in developing countries [36]. The adoption and implementation of tobacco control measures have strengthened the position of public health advocates against pressure from multinational tobacco companies [36].

One of the lessons learned from tobacco control is the need to focus on the upstream factors in the NCD pandemics such as multinational companies and globalized advertising and promotion of unhealthy products. Tobacco control activity at the global level is affected by the actions of the tobacco industry, increasing globalization of aggressive marketing, and changing social norms regarding smoking. As a result, it is difficult for any country to control tobacco use within its borders and a collective response is necessary. Policies focused on increasing price, decreasing access, restricting 


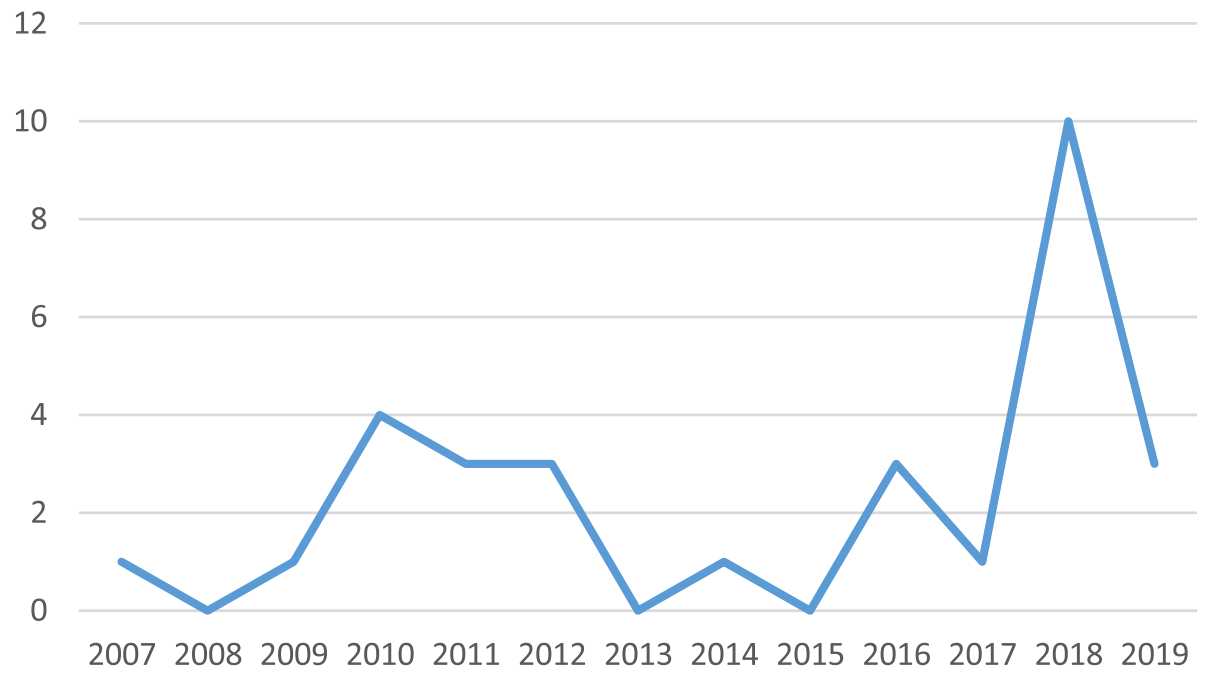

Fig. 2 Frequency distribution of studies on GHD for NCDs

advertising and improving labeling could be applied to products that promote NCDs, especially alcohol and processed foods that are high in sugar and fat [41].

The complexity and costs associated with effective management of NCDs require the involvement of a diverse group of actors. Given the increased pressure faced by developing countries with unprepared systems and economies, diverse partnerships may be a more important component in effective prevention and management of NCDs [30]. GHD researchers have studied international negotiations as a "two-level game" at national and international levels. At the international level, governments seek to maximize their ability to meet domestic pressures, while minimizing the negative consequences of foreign development. At the national level, domestic groups pressure the government to adopt favorable policies for their interests, and politicians seek power by building coalitions among those groups [62]. There is power asymmetry in international negotiations. This is exacerbated in the context of NCDs and GHD since health ministries and agencies are often less powerful within their governments. Two strategies to overcome this challenge are building coalitions and preparing for negotiation [30].

Governments must be the key stakeholders in policy development and provide leadership for implementation, monitoring, and evaluation through a multi-stakeholder platform. The government may decide to cooperate with other sectors and stakeholders, but it is in the best position to set the direction and overall strategy for achieving public health goals [63]. There are differing views within the government about the specific positions and strategies the country should take during negotiations. Creating an inter-ministerial committee is a useful way of using these different perspectives in international negotiations. The history of health-related trade negotiations indicates the importance of these institutional mechanisms for intersectoral collaboration [64]. After setting a clear negotiating agenda at the national level, the most conventional form of GHD takes place, i.e. formal negotiations between parties. In international negotiations, parties with different preferences need to exchange concessions. One party concedes to the preferences of the other party and, in exchange, ensures that its priority is reflected in the final text. In these negotiations, building coalitions with like-minded parties is a useful strategy for smaller countries that seek to achieve their objectives [30].

In the context of NCDs, a key aspect of GHD is the variety of nonstate actors that must be engaged with at different parts of the process, and it is crucial to understand the incentives of these actors pertaining to the costs and benefits of participation [34, 65, 66]. Civil society organizations must be involved in international negotiations on public health issues and must focus on providing and sharing relevant scientific information with national delegates. All the delegates and FCA participants in the FCTC argued that, at the beginning of the Intergovernmental Negotiating Body (INB) sessions, there were significant differences in the knowledge of national delegates of tobacco use and tobacco control. The FCA has helped close this gap by providing and sharing information with the delegates [37]. It is the actors involved in a negotiation process that determine whether to place an issue on the national and international agenda and whether to create alternatives for its effectiveness. It is thus necessary for NCD stakeholders to come 
Table 2 Analytical framework for examining negotiations

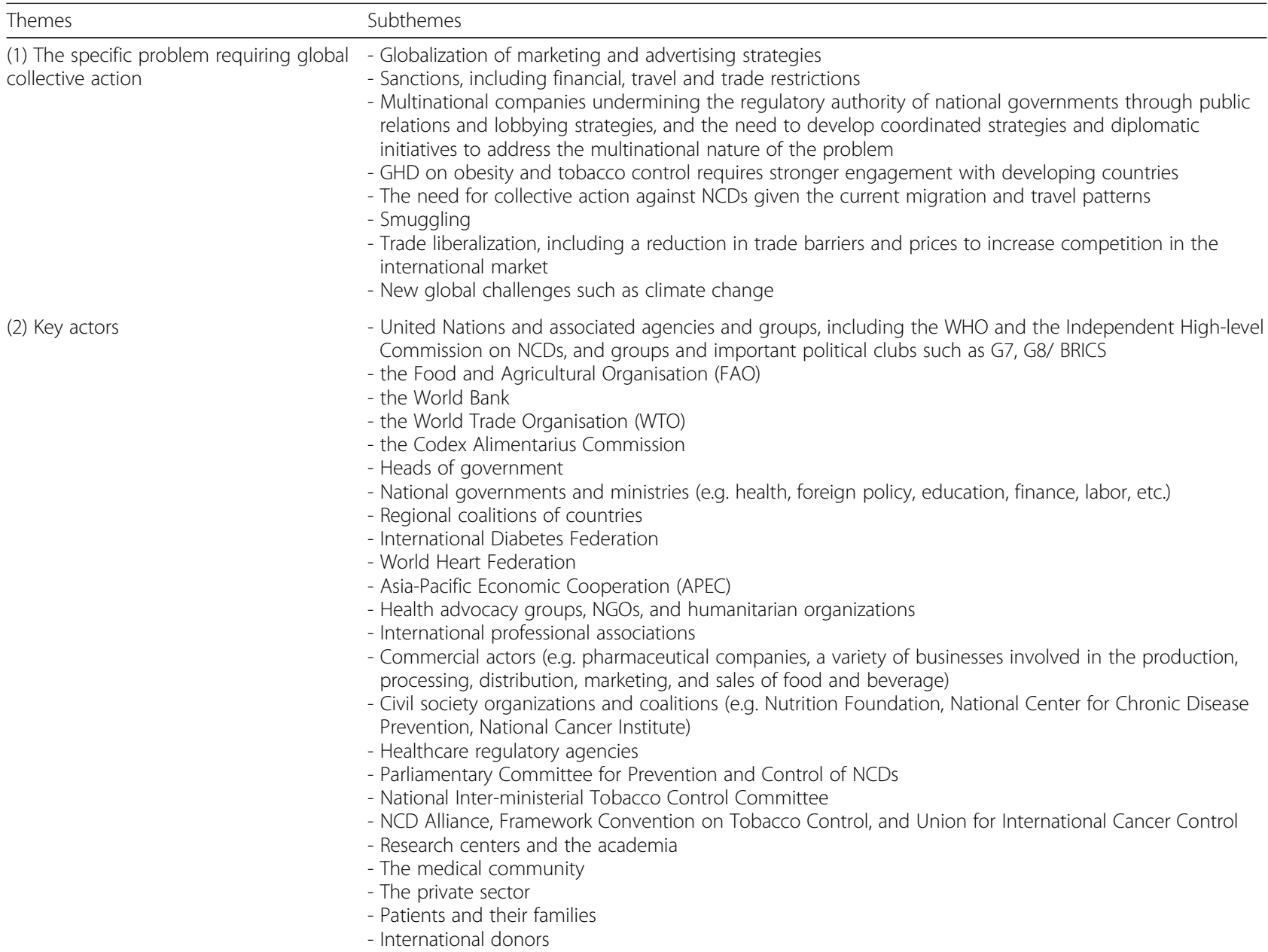

(3) Actors' interests in the problem

(4) Potential negotiation process

(5) Potential scenarios for collective action
- UN and WHO: technical support, non-binding recommendations and policy advice, international legal obligations and laws, advocacy

- Groups and political clubs such as G7, G8/ BRICS: Supporting reforms, develop framwork for regulation, support growth and development across globe, Strengthen international cooperation

- Governmental actors: governments represent power in diplomacy, while NGOs represent ideas and knowledge

- NGOs: monitoring, lobbying, brokering knowledge, offering technical expertise, and fostering inclusion; NGOs act as a catalyst to the process of developing policies and drive it by including stakeholders, offering technical expertise, and providing financial assistance; multisectoral partnerships aimed at capacity-building and strengthening health systems

- Industrial actors: reduced market share and profits due to measures to limit tobacco use

- Unions: advocacy of NCD Alliance to trigger a global policy response, the role of Framework Convention Alliance (FCA) in agenda-setting for tobacco control

- Policy champions: strong supportive role

- Scientific and technical capacity building

- Preparation for negotiations, mobilization of civil society organizations, dialogue with industry, consultation with experts, and sharing of information among national health agencies

- Entering the negotiating forum

- Monitoring, lobbying, brokering knowledge, offering technical expertise, and fostering inclusion

- Drawing attention to issues that are not yet on the GHD agenda or are largely overlooked

- Political leadership, strong mobilization, and advocacy from well-organized groups globally are crucial in triggering and sustaining a global policy response such as an international treaty

- A monitoring role that involves publicizing the diplomatic process 
Table 3 Prevention and control of NCDs at different levels of the GHD Pyramid

\begin{tabular}{|c|c|c|c|c|}
\hline & Actor & Tool (Process) & Content & Context \\
\hline $\begin{array}{l}\text { Core } \\
\text { Diplomacy }\end{array}$ & $\begin{array}{l}\text { - UN } \\
\text { - WHO } \\
\text { - the Food and } \\
\text { Agricultural } \\
\text { Organisation (FAO) } \\
\text { - the World Bank } \\
\text { - the World Trade } \\
\text { Organisation (WTO) } \\
\text { - the Codex } \\
\text { Alimentarius } \\
\text { Commission } \\
\text { - Independent High- } \\
\text { level Commission on } \\
\text { NCDs } \\
\text { - Heads of government } \\
\text { - International } \\
\text { professional } \\
\text { associations } \\
\text { - International Diabetes } \\
\text { Federation } \\
\text { - World Heart } \\
\text { Federation } \\
\text { - Asia-Pacific Economic } \\
\text { Cooperation (APEC) } \\
\text { - The Caribbean } \\
\text { Community } \\
\text { (CARICOM) }\end{array}$ & $\begin{array}{l}\text { - Policy brief } \\
\text { - Technical support } \\
\text { - Exchange of experiences } \\
\text { at the international level } \\
\text { - Knowledge generation } \\
\text { International legal } \\
\text { obligations and laws } \\
\text { - non-binding recommen- } \\
\text { dations and policy } \\
\text { advice } \\
\text { - advocacy }\end{array}$ & $\begin{array}{l}\text { - UN High-Level Meeting on } \\
\text { Prevention and Control of } \\
\text { NCDs } \\
\text { - WHO Global NCD Action } \\
\text { Plan } \\
\text { - WHO Global Monitoring } \\
\text { Framework for NCDs } \\
\text { - Recognition of NCDs as a } \\
\text { major challenge to } \\
\text { sustainable development } \\
\text { - Alliances, treaties, and other } \\
\text { agreements } \\
\text { - FCTC } \\
\text { - Regional Action Plan for } \\
\text { Prevention and Control of } \\
\text { NCDs } \\
\text { - Port of Spain Summit } \\
\text { Declaration "Uniting to Stop } \\
\text { the Epidemic of Chronic } \\
\text { NCDs" } \\
\text { - WHO Global Strategy on } \\
\text { Diet, Physical Activity and } \\
\text { Health } \\
\text { - Global Code of Practice on } \\
\text { the Marketing of Unhealthy } \\
\text { Food and Beverages to } \\
\text { Children } \\
\text { - Resolutions WHA63.14: } \\
\text { Marketing of Foods and } \\
\text { Non-alcoholic Beverages to } \\
\text { Children } \\
\text { - MPOWER Package } \\
\text { - Best Buys } \\
\text { - 2017 WHO Cancer } \\
\text { Resolution } \\
\text { - WHO Mental Health Action } \\
\text { Plan } \\
\text { - Montevideo } \\
\text { Roadmap 2018-2030 on } \\
\text { NCDs as a Sustainable } \\
\text { Development Priority } \\
\text { - The Paris Declaration on Aid } \\
\text { Effectiveness }\end{array}$ & $\begin{array}{l}\text { - Need for network formation at regional and } \\
\text { international levels } \\
\text { - Lack of resources at national and international } \\
\text { levels } \\
\text { - Effect of the political will of politicians } \\
\text { - The instrumental use of GHD to achieve other } \\
\text { foreign policy and diplomatic goals } \\
\text { - Differences in wealth, incidence and prevalence } \\
\text { of NCDs, and method of disease control }\end{array}$ \\
\hline $\begin{array}{l}\text { Multi- } \\
\text { stakeholder } \\
\text { diplomacy }\end{array}$ & $\begin{array}{l}\text { - Ministry of Health } \\
\text { - Ministry of Industry } \\
\text { - Ministry of Agriculture } \\
\text { - Ministry of Education } \\
\text { - Ministry of Urban } \\
\text { Development } \\
\text { - Ministry of Sports } \\
\text { - Environmental } \\
\text { organizations } \\
\text { - Media } \\
\text { - The academia } \\
\text { - Commercial actors } \\
\text { - Civil society } \\
\text { organizations and } \\
\text { coalition } \\
\text { - Healthcare regulatory } \\
\text { agencies } \\
\text { - Nutrition Foundation } \\
\text { - National Center for } \\
\text { Chronic Disease } \\
\text { Prevention, National } \\
\text { Cancer Institute } \\
\text { - Parliamentary } \\
\text { Committee for }\end{array}$ & $\begin{array}{l}\text { - Advocacy } \\
\text { - Scientific research } \\
\text { - Health in All Policies } \\
\text { - Clear guidelines to } \\
\text { strengthen coordination } \\
\text { mechanisms } \\
\text { - Whole-of-government } \\
\text { approach } \\
\text { - Publication of a } \\
\text { newsletter, shaming, and } \\
\text { symbolism } \\
\text { - Media advocacy to } \\
\text { influence policy } \\
\text { positions of countries } \\
\text { during negotiations } \\
\text { - Holding scientific } \\
\text { conferences }\end{array}$ & $\begin{array}{l}\text { - National Document on } \\
\text { Prevention and Control of } \\
\text { NCDs } \\
\text { - Agreements between } \\
\text { ministries } \\
\text { - National Plan for Tobacco } \\
\text { Control } \\
\text { - Development plans }\end{array}$ & $\begin{array}{l}\text { - Advocacy varies across different governments } \\
\text { and ministries (different cultures) } \\
\text { - Programs developed to change systems and } \\
\text { cultures (e.g. lifestyle changes) require } \\
\text { sustainable medical and political leadership } \\
\text { - A single plan is not responsive for all countries } \\
\text { and different contexts } \\
\text { - Significant difference between low-income } \\
\text { countries and the global context } \\
\text { - Political obstacles, including sanctions } \\
\text { - Geographical location, communication, and } \\
\text { health infrastructure } \\
\text { - Language barriers and the need to translate } \\
\text { tools to native languages } \\
\text { - Health-related socioeconomic factors are not } \\
\text { limited to the public health sector alone and } \\
\text { exist in other sectors of the health system, } \\
\text { highlighting the need for multi-sectoral action } \\
\text { - Poverty and social factors affecting health } \\
\text { - Implementing changes for cancer control that } \\
\text { are tailored to a specific context, society, and } \\
\text { culture } \\
\text { - NCDs threaten economic and human } \\
\text { development }\end{array}$ \\
\hline
\end{tabular}


Table 3 Prevention and control of NCDs at different levels of the GHD Pyramid (Continued)

\begin{tabular}{|c|c|c|c|c|}
\hline & Actor & Tool (Process) & Content & Context \\
\hline & $\begin{array}{l}\text { Control of NCDs } \\
\text { - National Inter- } \\
\text { ministerial Tobacco } \\
\text { Control Committee }\end{array}$ & & & \\
\hline $\begin{array}{l}\text { Informal } \\
\text { Diplomacy }\end{array}$ & $\begin{array}{l}\text { - NCD Alliance } \\
\text { - Research centers and } \\
\text { academic associations } \\
\text { - The medical } \\
\text { community } \\
\text { - The private sector } \\
\text { - Patients and their } \\
\text { families } \\
\text { - International donors } \\
\text { - NGOs } \\
\text { - Health advocacy } \\
\text { groups, NGOs, and } \\
\text { humanitarian } \\
\text { organizations } \\
\text { - National Cancer } \\
\text { Institute }\end{array}$ & $\begin{array}{l}\text { - Media } \\
\text { - Campaigns against } \\
\text { alcohol and tobacco use } \\
\text { - National Campaign for } \\
\text { Tobacco Control and } \\
\text { Smoking Cessation } \\
\text { - Specific activities such as } \\
\text { games for the elderly } \\
\text { - World No Tobacco Day }\end{array}$ & - NCD Advocacy Document & $\begin{array}{l}\text { - Understanding the historical context of } \\
\text { negotiations } \\
\text { - Any country is several markets rather than one, } \\
\text { with a wide variety of marketing types }\end{array}$ \\
\hline
\end{tabular}

together within and among countries to advocate for their needs and priorities [21].

Six key sub-themes were identified for the theme "actors' interests in the problem". WHO recommendations are non-binding, but represent the organization's official policy and reflect its norms and standards. Moreover, these recommendations indicate successful GHD. However, successful international agreements are not always implemented at a national level, and thus, they may not bring about the desired health benefits [40]. A weakness of the UN Declaration on the High-Level Meeting on NCDs in September 16, 2011, is that it was lacking in targets, funds, and activities. An important determinant in the relatively weak outcome of this exercise in GHD is the weak presence of advocacy groups and activists regarding NCDs. An important lesson from successful GHD in the past is the key role of civil society organizations in ensuring a strong collective response. This factor was critical to the success of FCTC negotiations [46].

It is necessary to strengthen governance and coordination structures across different sectors and levels to ensure that all relevant sectors are involved in NCD prevention measures. Countries must use a strong advocacy and communications strategy on multi-sectoral action for NCD prevention in order to increase NCD awareness among various sectors and resolve conflicts. Sustainable joint financing mechanisms are needed for the effective implementation of these actions. In addition, countermeasures must be employed to prevent industries from obstructing the implementation of NCD prevention measures [42]. Conflict of interest can hinder or halt policy development or participation of different sectors. One of the negative effects of actors' interests is industry interference with the process of policy development and implementation, especially the tobacco industry, which has interfered with the policy process in almost every country in LMIC. Market forces contribute to an increase in NCDs. For example, pandemics related to obesity and tobacco and alcohol use are mainly due to the successful marketing of unhealthy products. This outcome is a serious justification for government intervention through regulatory and legal responses.

NCD control requires political champions for advocacy. The most important outcome of the new diplomatic attention to NCDs is the platform provided for greater coordination on NCDs among the UN, national governments, and civil society. One of the key challenges in NCD prevention and control is the complexity of these diseases, as they are caused by a variety of risk factors and are associated with various agents, from the international level (e.g. multinational fast food companies) to the local level (e.g. unwalkable streets). In addition, not all NCDs are preventable. Due to these complexities, it is difficult to specify their targets and funds. The global response to NCDs must focus on generating multisectoral evidence about the transnational factors that contribute to the rise in NCDs and the potential impact of policies proposed to control them [41].

As for the theme "the potential negotiation process", four sub-themes were identified, including scientific and technical capacity building, preparation for negotiation, entering the negotiating forum, and monitoring, lobbying, brokering knowledge, offering technical expertise, and fostering inclusion. GHD is a political process based on the intersection of health, foreign policy, and trade that can take place at bilateral and multilateral levels through political negotiations $[66,67]$.

Therefore, it is first necessary to strengthen the scientific and technical capacities of health attachés and develop diplomats for the GHD platform. Preparation for 
negotiations by mobilization of civil society organizations, dialogue with industry, consultation with experts, and sharing of information among national health agencies are crucial steps before entering negotiations [36]. Moreover, to address many global public health issues in which some countries put political expediencies and their interests above collective global interests, one strategy is to hold countries accountable by publicizing their positions and reward positions that support a strong treaty [37]. GHD always takes place through national politics. Therefore, the successful conclusion of international negotiations is not the end, but part of a process and GHD must be conducted and evaluated by considering the barriers and issues concerning national action [40].

Three sub-themes were identified for the theme "potential scenarios for collective action": First scenario: Drawing attention to issues that are not yet on the GHD agenda or are largely overlooked. GHD is the process of negotiated collective action for global health that can eventually lead to new forms of global health policy and governance for tackling global health challenges. It is clear that the development of national policies and FCTC negotiations have clearly interacted. Once Canada adopted large graphic health warnings, Thailand, Brazil, and the European Union followed suit [68]. Until recently, the field of GHD on NCDs was primarily WHOcentric. A key challenge was to bring NCDs beyond the traditional health forum and that is why the UN HighLevel Meeting is regarded as a great opportunity. When deciding to engage in GHD for a problem identified during the agenda-setting phase, an important consideration for policymakers is the impact of the media on the political agenda. The media is deemed to play a greater role in putting issues on the foreign policy agenda than the domestic policy agenda [69].

Second scenario: Political leadership, strong mobilization, and advocacy from well-organized groups globally are crucial in triggering and sustaining a global policy response such as an international treaty. Success in tackling the NCD crisis depends on strong and effective leadership. A strong leader can act as a catalyst and bring about change [70]. Moreover, NGOs and civil society have provided significant support for policy development and the interaction of various actors. NGOs can act as a catalyst to trigger the policy process and drive this process by coordinating the entry of stakeholders, offering technical support, and providing financial assistance. In addition, coalitions and networks may be formed ton ensure that implementation of certain policies will continue. These networks tend to engage various sectors in this process. Professional associations can also become part of advocacy and coalition networks [41].
Third scenario: A monitoring role that involves publicizing the diplomatic process, such as exposing the position of countries in documents distributed to diplomats and on the Internet for the general public. There is an established Monitoring and Evaluation for Port of Spain declaration which is robust and no other NCD policy has such a mechanism on a periodic basis. Monitoring of the Declaration was cunducted by CARICOM and the Pan American Health Organization (PAHO) and evaluating was cunducted by the University of the West Indies $[67,71]$.There is an urgent need for advocacy to raise NCD awareness at the national and international levels as a development problem, not just a health problem. In addition, increasing the capacity for policy research and implementation is needed in all countries [46].

\section{Conclusion}

This paper has highlighted the role of global health diplomacy in all three levels for preventing and controlling of NCDs. There is an urgent need for advocacy to raise NCD awareness at the national and international levels as a development problem, not just a health problem. In addition, increasing the capacity for policy research and implementation is needed in all countries. The development and adoption of a global policy to tackle the rise in chronic diseases in developing and developed countries requires policymakers that engage in GHD. Successful developments in global health policy depend on the performance and respectful relationships of stakeholders, and global health diplomats need to have an understanding of the complexities of institutional structures and the functional relationships among international institutions involved in health. A successful and sustainable plan for tacking NCDs entails partnerships among national governments, the private sector, and civil society at international, national, and local levels.

\section{Abbreviations}

NCDs: Noncommunicable diseases; GHD: Global health diplomacy; UN: United Nations; WHO: World Health Organization; LMIC: Low and middle-income countries; NGOs: Non-governmental organizations;

APEC: Asia-Pacific Economic Cooperation; PAHO: Pan American Health Organization; CARICOM: The Caribbean Community; FCTC: Framework Convention on Tobacco Control; NCDA: Noncommunicable diseases alliance; SDGs: Sustainable development goals

\section{Acknowledgements}

Not applicable.

\section{Authors' contributions}

MA, MAL and AAT designed the research:: MA, MAL and AAT conducted it; $M A$ and AAT extracted the data; and: MA, MAL and AAT and MM wrote the paper. AAT had primary responsibility for final content. All authors read and approved the final manuscript.

\section{Funding}

M Afshari conducted this study with research funding from Iran University of Medical Science (97-3-37-12777). The authors have no other relevant 
affiliations or financial involvement with any organization or entity with a financial interest in or financial conflict with subject matter or materials discussed in the manuscript.

\section{Availability of data and materials}

Not applicable.

\section{Ethics approval and consent to participate}

Not applicable.

\section{Consent for publication}

Not applicable.

\section{Competing interests}

The authors declare that they have no competing interests.

\section{Author details}

${ }^{1}$ Department of Health Service Management, School of Health Management and Information Sciences, Iran University of Medical Sciences, Tehran, Iran. ${ }^{2}$ Department of Epidemiology, School of Public Health, Iran University of Medical Sciences, Tehran, Iran.

Received: 6 January 2020 Accepted: 22 April 2020

Published online: 06 May 2020

\section{References}

1. Fidler D, Drager N. Global Health and Foreign Policy: strategic opportunities and challenges. Background Paper for the Secretary-General's Report on Global Health and Foreign Policy: World Health Organization Disponivel em; 2009. http://www.who.int/trade/events/UNGA_Background_Rep3_2.pdf. Accessed 11 May 2009

2. Fidler DP. The challenges of global health governance: Council on Foreign Relations, Incorporated; 2010.

3. Garrett L. The challenge of global health. Foreign Affairs. 2007;86(1):14-38

4. Fidler D. Pathways for global health diplomacy: Perspectives on health in foreign policy. In: Globalization, trade and health working paper series 2008; 2008

5. United Nations General Assembly. Resolution adopted by the General Assembly. 63/33. In: Global health and foreign policy; 2008.

6. United Nations General Assembly. Resolution adopted by the General Assembly. 64/108. In: Global health and foreign policy; 2009.

7. United Nations General Assembly. Resolution adopted by the Genera; Assembly. 65/95. In: Global health and foreign policy; 2010

8. Fidler D. Navigating the Global Health Terrain: Preliminary Considerations on Mapping Global Health Diplomacy. In: World Health Organization Globalization, Trade, and Health Working Paper Series; 2008.

9. Drager N, Fidler DP. Foreign policy, trade and health: at the cutting edge of global health diplomacy. Bull World Health Organ. 2007:85(3):162.

10. Kickbusch I. Global health diplomacy: how foreign policy can influence health. BMJ. 2011;342:d3154. https://doi.org/10.1136/bmj.d3154.

11. Kickbusch I, Novotny TE, Drager N, Silberschmidt G, Alcazar S. Global health diplomacy: training across disciplines. Bull World Health Organ. 2007;85(12): 971-3.

12. Kickbusch I, Silberschmidt G, Buss P. Global health diplomacy: the need for new perspectives, strategic approaches and skills in global health. Bull World Health Organ. 2007:85(3):230-2.

13. Lee K, Smith R. What is 'global health diplomacy'? A conceptual review; 2011.

14. Katz R, Kornblet S, Arnold G, Lief E, Fischer JE. Defining health diplomacy: changing demands in the era of globalization. Milbank Q. 2011;89(3):50323.

15. Brown MD, Mackey TK, Shapiro CN, Kolker J, Novotny TE. Bridging public health and foreign affairs: the tradecraft of global health diplomacy and the role of health attachés. Sci Diplomacy. 2014;3(3):1-12.

16. Suleman M, Ali R, Kerr DJ. Health diplomacy: a new approach to the Muslim world? Glob Health. 2014;10:50

17. Anonymous. The global alliance for chronic diseases. Lancet (London, England). 2009:373(9681):2084

18. WHO Key facts. Noncommunicable diseases. 2018.

19. Lozano R, Naghavi M, Foreman K, Lim S, Shibuya K, Aboyans V, et al. Global and regional mortality from 235 causes of death for 20 age groups in 1990 and 2010: a systematic analysis for the global burden of disease study 2010 Lancet. 2012:380(9859):2095-128. https://doi.org/10.1016/S01406736(12)61728-0.

20. Naghavi M, Wang H, Lozano R, Davis A, Liang X, Zhou M, Vollset SE, et al. Global, regional, and National age-sex Specific all-Cause and cause-specific mortality for 240 causes of death, 1990-2013: a systematic analysis for the global burden of disease study 2013. Lancet. 2015;385(9963):117-71. https:// doi.org/10.1016/S0140-6736(14)61682-2.

21. Pearlman PC, Vinson C, Singh T, Stevens LM, Kostelecky B. Multi-stakeholder partnerships: breaking down barriers to effective cancer-control planning and implementation in low-and middle-income countries. Sci Dipl. 2016;5: $1-15$.

22. United Nations General Assembly, Resolution 66/2, "Political Declaration of the High-Level Meeting of the General Assembly on the Prevention and Control of Non-communicable Diseases," September 19, 2011, http://www. who.int/.nmh/events/un_ncd_summit2011/political_declaration_en.pdf.

23. World Health Organization. Global Action Plan for the Prevention and Control of Noncommunicable Diseases, 2013-2020. Geneva: WHO Press, 2013.

24. House W. Follow-up to the political declaration of the high-level meeting of the general assembly on the prevention and control of non-communicable diseases. World Health Organization; 2013. WHA66.10.

25. "Draft Comprehensive Global Monitoring Framework and Targets for the Prevention and Control of Noncommunicable Diseases," A66/8. World Health Organization; 2013. http://apps.who.int/gb/ebwha/pdf_files/WHA66/ A66 8-en.pdf. Accessed 20 Mar 2013.

26. United Nations General Assembly, Draft Resolution A/69/L.85, "Draft Outcome Document of the United Nations Summit for the Adoption of the Post-2015 Development,". 2015. http://www.un.org/ga/search/view_doc asp?symbol=A/69/L.85\&Lang=E. Accessed 22 May 2018.

27. Gómez EJ. The politics of global health diplomacy: conceptual, theoretical, and empirical lessons from the United States, Southeast Asia, and Latin America. Global HIV/AIDS Politics, Policy, and Activism: Persistent Challenges and Emerging Issues [3 volumes]: Persistent Challenges and Emerging Issues. 2013. p. 73-90.

28. Mackey TK. Global health diplomacy and the governance of counterfeit medicines: a mapping exercise of institutional approaches. J Health Diplomacy. 2013;1(1):1-20.

29. Hawkes N. Ebola outbreak is a public health emergency of international concern, WHO warns. BMJ. 2014. https://doi.org/10.1136/bmj.g5089.

30. Blouin C. Global responses to chronic diseases: what lessons can political science offer? Adm Sc. 2012;2(1):120-34.

31. Liberati A, Altman DG, Tetzlaff J, Mulrow C, Gotzsche PC, loannidis JP, et al. The PRISMA statement for reporting systematic reviews and meta-analyses of studies that evaluate healthcare interventions: explanation and elaboration. BMJ. 2009. https://doi.org/10.1136/bmj.b2700.

32. Mitton C, Adair CE, McKenzie E, Patten SB, Perry BW. Knowledge transfer and exchange: review and synthesis of the literature. Milbank Q. 2007 https://doi.org/10.1111/j.1468-0009.2007.00506.x.

33. Hospedales CJ, Samuels TA, Cummings R, Gollop G, Greene E. Raising the priority of chronic noncommunicable diseases in the Caribbean. Rev Panam Salud Publica. 2011;30(4):393-400.

34. Lencucha $\mathrm{R}$, Kothari $\mathrm{A}$, Labonté $\mathrm{R}$. The role of non-governmental organizations in global health diplomacy: negotiating the framework convention on tobacco control. Health Policy Plan. 2010;26(5):405-12.

35. Lee K, Chagas LC, Novotny TE. Brazil and the framework convention on tobacco control: Global Health diplomacy as soft power. PLoS Med. 2010; 7(4):e1000232. https://doi.org/10.1371/journal.pmed.1000232.

36. Blouin C, Dubé L. Global health diplomacy for obesity prevention: lessons from tobacco control. J Public Health Policy. 2010:31(2):244-55.

37. Mamudu HM, Glantz SA. Civil society and the negotiation of the framework convention on tobacco control. Global public health. 2009; $4(2): 150-68$

38. Teixeira LA, Paiva CH, Ferreira VN. The World Health Organization framework convention on tobacco control in the Brazilian political agenda, 2003-2005. Cadernos de saude publica. 2017S. https://doi.org/10.1590/0102311 X00121016.

39. Sener SF. Has medical diplomacy reached an inflection point? Am J Surg. 2014;207(3):315-20.

40. Smith $\mathrm{R}$, Irwin R. Measuring success in global health diplomacy: lessons from marketing food to children in India. Glob Health. 2016;12(1):28. 
41. Wipfli HL, Samet JM. Moving beyond global tobacco control to global disease control. Tobacco Contro. 2012;21(2):269-72.

42. Juma PA, Mapa-Tassou C, Mohamed SF, Mwagomba BL, Ndinda C, Oluwasanu M, Mbanya JC, Nkhata MJ, Asiki G, Kyobutungi C. Multi-sectoral action in non-communicable disease prevention policy development in five African countries. BMC Public Health. 2018:18(1):953.

43. Dain K. Challenges facing global health networks: the NCD Alliance experience: Comment on "Four challenges that global health networks face". Int J Health Policy Manag. 2018;7(3):282-5. https://doi.org/10.15171/ ijhpm.2017.93.

44. Russell A, Wainwright M, Tilson M. Means and ENDS- e-cigarettes, the framework convention on tobacco control, and global health diplomacy in action. Global Public Health. 2018;13(1):83-98. https://doi.org/10.1080/ 17441692.2016.1152284.

45. Frech S, Muha CA, Stevens LM, Trimble EL, Brew R, Perin DP, Luciani S, Mohar A, Piñeros M, Vidaurre T, Morgan DR. Perspectives on strengthening Cancer research and control in Latin America through partnerships and diplomacy: experience of the National Cancer Institute's Center for Global Health. J Global Oncol. 2018;4:1-1.

46. Beaglehole R, Bonita R, Horton R, Adams C, Alleyne G, Asaria P, Baugh V, Bekedam H, Billo N, Casswell S, et al. Priority actions for the noncommunicable disease crisis. Lancet. 2011;377:1438-47.

47. Nishtar S, Niinistö S, Sirisena M, Vázquez T, Skvortsova V, Rubinstein A, Mogae FG, Mattila P, Ghazizadeh HS, Kariuki S, Narro RJ. Time to deliver: report of the WHO Independent High-Level Commission on NCDs. Lancet (London, England). 2018;392(10143):245.

48. Samuels TA, Hospedales CJ. From Port-Of-Spain summit to United Nations high level meeting: CARICOM and the global non-communicable disease agenda. West Indian Med J. 2011;60(4):387-91.

49. Hatefi A, Allen LN, Bollyky TJ, Roache SA, Nugent R. Global susceptibility and response to noncommunicable diseases. Bull World Health Organ. 2018; 96(8):586.

50. Wickramasinghe K, Wilkins E, Foster C, Fadhil I, Hammerich A, Slama S, El Berri H, Elimam M, Adib S, Al-Mudwahi M, Townsend N. The development of national multisectoral action plans for the prevention and control of noncommunicable diseases: experiences of national-level stakeholders in four countries. Glob Health Action. 2018;11(1):1532632.

51. Mendis $\mathrm{S}$. The policy agenda for prevention and control of noncommunicable diseases. Br Med Bull. 2010;96(1):23-43.

52. Maher A, Sridhar D. Political priority in the global fight against noncommunicable diseases. J Global Health. 2012. https://doi.org/10.7189/jogh. 02.020403.

53. Gneiting U, Schmitz HP. Comparing global alcohol and tobacco control efforts: Network formation and evolution in international health governance. Health Policy Plann. 2016;31(suppl_1):i98-109.

54. Magnusson RS. Non-communicable diseases and global health governance: enhancing global processes to improve health development. Glob Health. 2007;3(1):2.

55. Battams S, Townsend B. Power asymmetries, policy incoherence and noncommunicable disease control-a qualitative study of policy actor views. Crit Public Health. 2019;29(5):596-609.

56. Kirton J, Knight WA, Hospedales CJ, Hippolyte D, Kulik J. Regional and global impacts of the 2007 Port-of-Spain Declaration on noncommunicable diseases. Rev Panam Salud Publica. 2018;42:e194. https://doi.org/10.26633/ rpsp.2018.194.

57. Greaves NS, Tull J, Hassell T, Samuels TA. Communication strategy to disseminate the results of the evaluation of the Port-of-Spain Declaration (POSDEVAL) across multiple audiences in the Caribbean. Rev Panam Salud Publica. 2018;42:e186. https://doi.org/10.26633/rpsp.2018.186.

58. Chattu VK, Knight AW. Port of Spain summit declaration as a successful outcome of global health diplomacy in the Caribbean region: a systematic review. Health Promotion Perspect. 2019;9(3):174.

59. McBride B, Hawkes S, Buse K. Soft power and global health: the sustainable development goals (SDGs) era health agendas of the G7, G20 and BRICS. BMC Public Health. 2019;19(1):815.

60. Collins TE, Nugent R, Webb D, Placella E, Evans T, Akinnawo A. Time to align: development cooperation for the prevention and control of noncommunicable diseases. BMJ. 2019;366:14499.

61. Collin J, Lee K, Bissel K. The framework convention on tobacco control: the politics of global health governance. Third World Q. 2002;23(2):265-82.
62. Putnam RD. Diplomacy and domestic politics: the logic of two-level games. Int Organ. 1988;42:427-60.

63. WHO. Set of Recommendations on the marketing of foods and nonalcoholic beverages to children. Geneva: World Health Organization; 2010.

64. Blouin C. Trade policy and health: from conflicting interests to policy coherence. Bull WHO. 2007;85:161-244.

65. Smith R. Global health governance and global public goods. In: Hein W, Drager N, editors. Making sense of Global Health governance: a policy perspective; BusemK. London: Palgrave MacMillan; 2009.

66. Kickbusch I, Rosskam E. Introduction: the art and practice of conducting global health negotiations in the 21st century. In: Negotiating and navigating global health: Case studies in global health diplomacy; 2012. p. $1-8$.

67. Chattu VK, Sakhamuri S. Port-Of-Spain declaration for global NCD prevention. Lancet. 2018;391(10131):1682. https://doi.org/10.1016/s01406736(18)30790-6.

68. Collin J, Lee K. Globalization and the politics of health governance: The framework convention on tobacco control. In: Cooper A, Kirton J, editors. Innovation in Global Health Governance: Critical Cases. Waterloo: Ashgate/ CIGl; 2009.

69. Wood D, Peake J. The dynamics of foreign policy agenda setting. Am Polit Sci Rev. 1998;92:173-84.

70. Donnelly J. How did Sierra Leone provide free health care? Lancet. 2011; 377:1393-6.

71. Samuels TA, Unwin N. The 2007 Caribbean Community Port-of-Spain Declaration on noncommunicable diseases: an overview of a multidisciplinary evaluation. Rev Panam Salud Publica. 2018;42:e193. https://doi.org/10.26633/RPSP.2018.193 PMID: 31093220; PMCID: PMC6385896.

\section{Publisher's Note}

Springer Nature remains neutral with regard to jurisdictional claims in published maps and institutional affiliations.

Ready to submit your research? Choose BMC and benefit from:

- fast, convenient online submission

- thorough peer review by experienced researchers in your field

- rapid publication on acceptance

- support for research data, including large and complex data types

- gold Open Access which fosters wider collaboration and increased citations

- maximum visibility for your research: over $100 \mathrm{M}$ website views per year

At BMC, research is always in progress.

Learn more biomedcentral.com/submissions 